\title{
TEE-Vorhersage und -Prävention bei Patienten mit Keimzelltumoren und Cisplatin-Therapie
}

\begin{abstract}
Patienten mit Keimzelltumoren (GCT) haben unter Cisplatin-basierter Chemotherapie ein erhöhtes Risiko für thromboembolische Ereignisse (TEE). Retrospektiv wurden nun die Rolle möglicher Prädiktoren und die Bedeutung der Prophylaxe mit niedermolekularen Heparinen (LMWH) untersucht.
\end{abstract}

\begin{abstract}
$\mathrm{n}$ einer früheren Studie hatten Patienten mit GCT unter Cisplatin-basierter Chemotherapie ein höheres TEE-Risiko als andere Tumorpatienten im gleichen Alter (relatives Risiko 3,4). Erhöhte Serum-Laktatdehydrogenase(LDH)-Werte und eine große Körperoberfläche schienen unabhängige Prädiktoren dafür zu sein. In der vorliegenden Studie sollte die Rolle prädiktiver Faktoren erneut geprüft und der Stellenwert der TEE-Prophylaxe bei GCT-Patienten bestimmt werden.
\end{abstract}

295 GCT-Patienten, die zwischen 2001 und 2014 in der Erstlinie eine Cisplatinbasierte Chemotherapie erhalten hatten, wurden retrospektiv analysiert. Ab 2005 wurde die Prophylaxe mit LMWH empfohlen und bei Patienten mit erhöhter LDH und/oder einer Körperoberfläche $>1,9 \mathrm{~m}^{2}$ zunehmend implementiert.

Zwischen 2001 und 2004 hatten 75 (Kohorte 1) und zwischen 2005 und 2014 204 Patienten (Kohorte 2) eine Chemotherapie mit Cisplatin $\left(100 \mathrm{mg} / \mathrm{m}^{2}\right) \mathrm{er}$ halten, das hauptsächlich mit Bleomycin und Etoposid kombiniert wurde. In der Gesamtgruppe kam es bei $14 \%$ der Patienten zu einem TEE (26 tiefe Venen[DVT], 2 arterielle Thrombosen und 10 oberflächliche Thrombophlebitiden). Eine DVT entwickelte sich bei 12,7\% der 204 Patienten mit Risikofaktoren gegenüber nur 2,6\% der 75 Betroffenen ohne
Risikofaktoren $(p=0,01)$. Die meisten superfiziellen Thrombophlebitiden betrafen den Arm, an dem die Chemotherapeutika infundiert wurden.

Nach zunehmender Beachtung des Prophylaxe-Protokolls wurde dieses in Kohorte 2 bei $68 \%$ der Patienten (104/200) angewandt. Die Häufigkeit von TEE (primärer Endpunkt der Studie) sank hier bei Risikopatienten durch die Gabe von LMWH auf nahezu die Hälfte (9,2 vs. $16,6 \%)$. Dies spricht somit erneut für die Prophylaxe mit LMWH bei Patienten mit GCT und erhöhtem Risiko.

Fazit: Patienten mit GCT haben unter Cisplatin-basierter Chemotherapie ein erhöhtes Risiko für TEE. Ein Voraussagefaktor ist ein erhöhter LDH-Wert. Die Prophylaxe mit LMWH führt zu einer erheblichen Reduktion der TEE bei GCT-Risikopatienten. Brigitte Schalhorn

Gizzi $\mathrm{M}$ et al. Predicting and preventing thromboembolic events in patients receiving cisplatin-based chemotherapy for germ cell tumours. Eur J Cancer. 2016;69:151-7.

\section{Hochrisiko-Seminom: Surveillance oder adjuvante Radiotherapie?}

\section{Die beste Therapiestrategie für Patienten mit Seminomen im klinischen Stadium I (CS-1) wird nach wie vor kontrovers diskutiert: adjuvante Radio- therapie (RT) oder Überwachung? Wovon profitieren Patienten, die mit einem Tumordurchmesser von $\geq 6 \mathrm{~cm}$ ein hohes Rezidivrisiko haben?}

\footnotetext{
n der retrospektiven Analyse wurden 473 Patienten mit CS-1-Seminomen mit einem Tumordurchmesser von $\geq 6 \mathrm{~cm}$ aus der Danish Testicular Cancer Database betrachtet. Zwischen 1987 und 2007 erhielten 254 von ihnen eine adjuvante RT, 219 wurden nur überwacht (u. a. mit CTs von Abdomen und Becken). Das mediane Follow-up umfasste 14 Jahre nach RT und 24 Jahre bei Surveillance. Im Rahmen der adjuvanten RT wurden auch paraaortale und ipsilaterale hohe IliakalLymphknoten mit Gesamtdosen von 24 26 Gy bestrahlt. Vor 1990 wurden nur $2 \%$ der Patienten bestrahlt, nach 1995 stieg der Anteil auf über $75 \%$ an.

Es zeigten sich keine signifikanten Unterschiede zwischen den Gruppen: Die 10-Jahres-Überlebensrate betrug in
}

der Beobachtungsgruppe 92,4\%, in der RT-Gruppe $94,2 \%$. Patienten mit adjuvanter RT erhielten insgesamt mehr Behandlungen als solche unter Surveillance, aber es fand sich kein signifikanter Unterschied in der Anzahl der Patienten mit mehr als einer Therapielinie. In der Surveillance-Gruppe erlitten deutlich mehr Patienten ein Rezidiv (nach 10 Jahren 32 vs. 2,8\%). Knapp ein Drittel dieser Patienten erhielt also noch eine tumorspezifische Therapie.

Sekundärtumoren traten in beiden Gruppen vergleichbar häufig auf $(6,1 \%$ in der Surveillance- bzw. 9,9\% in der RT-Gruppe) und umfassten ein breites Spektrum von Lokalisationen. In beiden Gruppen verstarben $33 \%$ der Patienten an ihren Sekundärtumoren. Da auch Pa- tienten ohne adjuvante RT bei engmaschiger Überwachung und gezielter Behandlung von Rezidiven eine Heilungsrate von nahezu $100 \%$ haben, stehen den Befürwortern der adjuvanten Therapie die Anhänger der alleinigen Überwachung gegenüber, die eine Übertherapie für gut zwei Drittel der Patienten vermeiden möchten. Die Forscher selbst empfehlen ihren Patienten nach wie vor die aktive Überwachung.

Fazit: Die 10-Jahres-Überlebensraten der Patienten mit CS-1-Seminomen und hohem Rezidivrisiko sind unabhängig von RT oder Surveillance gleich hoch. Die adjuvante RT reduziert effektiv das Rezidivrisiko, bedeutet aber bei etwa zwei Drittel der Patienten eine Übertherapie. Brigitte Schalhorn

Mortensen MS et al. Surveillance versus adjuvant radiotherapy for patients with high-risk stage I seminoma. Cancer. 2017;123(7):1212-8. 